\title{
The Involvement of the csy1 Gene in the Antimicrobial Resistance of Acinetobacter baumannii
}

\author{
Tingting Guo ${ }^{1,2,3}$, Xiaoli Sun ${ }^{1}$, Mengying Li ${ }^{1,4}$, Yuhang Wang ${ }^{1}$, Hongmei Jiao ${ }^{1}$ and \\ Guocai $\mathrm{Li}^{1,2,3 *}$ \\ ${ }^{1}$ Department of Microbiology, School of Medicine, Yangzhou University, Yangzhou, China, ${ }^{2}$ Jiangsu Key Laboratory of \\ Zoonosis/Jiangsu Co-Innovation Center for Prevention and Control of Important Animal Infectious Diseases and Zoonoses, \\ Yangzhou University, Yangzhou, China, ${ }^{3}$ Jiangsu Key Laboratory of Integrated Traditional Chinese and Western Medicine for \\ Prevention and Treatment of Senile Diseases, Yangzhou, China, ${ }^{4}$ Department of Pharmacy, Suzhou Hospital of Integrated \\ Traditional Chinese and Western Medicine, Suzhou, China
}

OPEN ACCESS

Edited by:

Jessica L. Jones, United States Food and Drug Administration, United States

Reviewed by: Prasanth Manohar,

Zhejiang University-University of Edinburgh Institute, China

Tingting $X u$,

Jinan University, China

*Correspondence:

Guocai Li

gcli@yzu.edu.cn

Specialty section:

This article was submitted to Infectious Diseases - Surveillance,

Prevention and Treatment,

a section of the journal

Frontiers in Medicine

Received: 18 October 2021

Accepted: 03 January 2022

Published: 26 January 2022

Citation:

Guo $T$, Sun X, Li M, Wang Y, Jiao $H$ and $\mathrm{Li} G$ (2022) The Involvement of the csy1 Gene in the Antimicrobial

Resistance of Acinetobacter baumannii. Front. Med. 9:797104 doi: 10.3389/fmed.2022.797104
Acinetobacter baumannii is an important, opportunistic nosocomial pathogen that causes a variety of nosocomial infections, and whose drug resistance rate has increased in recent years. The CRISPR-Cas system exists in several bacteria, providing adaptive immunity to foreign nucleic acid invasion. This study explores whether CRISPR-Cas is related to drug resistance. Antibiotics were used to treat strains ATCC19606 and AB43, and the expression of CRISPR-related genes was found to be changed. The Csy proteins (Csy $1-4)$ were previously detected to promote target recognition; however, the potential function of csy1 gene is still unknown. Thus, the Rec $\mathrm{Ab}$ homologous recombination system was utilized to knock out the csy1 gene from A. baumannii AB43, which carries the Type I-Fb CRISPR-Cas system, and to observe the drug resistance changes in wild-type and csy1-deleted strains. The AB43 $\Delta$ csy 1 mutant strain was found to become resistant to antibiotics, while the wild-type strain was sensitive to antibiotics. Moreover, transcriptome analysis revealed that the csy 1 gene regulates genes encoding CRISPR-Cas-related proteins, drug-resistant efflux pumps, membrane proteins, and oxidative phosphorylation-related proteins, inhibiting antimicrobial resistance in $A$. baumannii. The in vitro resistance development assay revealed that the complete CRISPR-Cas system could inhibit the development of bacterial resistance. Our findings expand our understanding of the role of CRISPR-Cas csy1 gene in A. baumannii and link the CRISPR-Cas system to the biogenesis of bacterial drug-resistant structures.

Keywords: Acinetobacter baumannii, nosocomial pathogen, CRISPR-Cas system, antimicrobial resistance, csy1 gene

\section{INTRODUCTION}

Acinetobacter baumannii, a non-fermented Gram-negative bacterium, is one of the primary causes of nosocomial infections worldwide. It mainly causes ventilator-associated pneumonia and blood, urinary tract, skin, and soft tissue infections, particularly in critically ill patients in the intensive care unit (1). Over the past few years, the drug resistance rate of $A$. baumannii has gradually increased (2). Due to the treatment challenges posed by emerging and increasing drug resistance, multi-drug resistant (MDR) A. baumannii poses a global threat to human health (3). 
The CRISPR-Cas system is an immune system used in prokaryotes resisting invasion of foreign genetic elements. It generally consists of three parts: a CRISPR array, a leader sequence, and Cas-related proteins (4). In general, three different stages have been described in the CRISPR-Cas immune response: (i) adaptation, (ii) CRISPR (Cr) RNA expression and maturation, and (iii) interference (5). The CRISPR-Cas system directs sequence-specific cleavage of phage and plasmid nucleic acids using nucleases programmed by small RNAs (6). The CRISPRCas system also inhibits conjugation and transformation, thereby limiting horizontal gene transfer. As the latter significantly affects bacterial evolution, the spread of antibiotic resistance and virulence determinants is the most pronounced (7). Several studies have demonstrated that the CRISPR-Cas system is associated with bacterial drug resistance. A previous study has demonstrated that a variety of genes are regulated by the Campylobacter jejuni II CRISPR-Cas9 system to promote bacterial drug resistance (8). The CRISPR/Cas9 system can mediate MDR Escherichia coli to restore antibiotic sensitivity (9). The Cas9-dependent CRISPR-Cas system of the intracellular bacterial pathogen Francisella novicida enhances antibiotic resistance by strengthening envelope integrity (10). In the I-F CRISPR-Cas system, multiple Cas proteins (Csy1-4) and CRISPR RNA (CrRNA) form a surveillance complex (Csy complex) for target recognition (11). Studies have shown that Csy proteins (Csy1-4) promote target recognition by enhancing sequencespecific hybridization between CRISPR RNA and complementary target sequences (12).

The Type I CRISPR-Cas system is the most widely distributed in nature (13). The unique feature of Type I-F CRISPR-Cas is the fusion of Cas 2 and Cas3 (Cas2/3), together with Cas 1 mediating the integration of the spacer into the CRISPR site $(14,15)$. There are two primary subtypes of the I-F CRISPR-Cas system known in A. baumannii, namely, Type I-Fa and Type I-Fb (16-18). The composition of Type I-Fa and Type I-Fb is illustrated in Figure 1. The Csyl protein is missing in the Type I-Fa CRISPR-Cas system; however, the other components contain additional domains that can compensate for the role of Csyl protein (19). Moreover, the role of csy1 gene in antimicrobial resistance is still unknown.

Our study aimed to research whether antibiotics affect the expression of CRISPR-Cas-related genes in A. baumannii to determine the relationship of csyl gene with antimicrobial resistance.

\section{MATERIALS AND METHODS}

\section{Bacterial Strains and Plasmids}

This study included standard strain A. baumannii ATCC19606 (GenBank: CP045108.1, https://www.ncbi.nlm.nih.gov/nuccore/ CP045108.1), which carries the complete CRISPR-Cas subtype I-Fa system, and clinical isolates of A. baumannii AB43 (GenBank: CP083182.1, https://www.ncbi.nlm.nih.gov/nuccore/ 2095284784), carrying the complete CRISPR-Cas subtype I$\mathrm{Fb}$ system. Moreover, AB133, ABF7, and ABE5 are clinical isolates containing an incomplete I-Fa system, an incomplete IFb system, or no CRISPR-Cas system, respectively (Table 1). The clinical isolates used in this study were isolated from the Affiliated Hospital of Yangzhou University and identified in our laboratory. The bacterial strains and plasmids used in this study are listed in Table 1.

CRISPR-Cas Finder (https://crisprcas.i2bc.paris-saclay.fr/ CrisprCasFinder/Index) was used to determine the existence of the CRISPR-Cas system and spacers in the genomes of strains ATCC19606 and AB43. The existence of clinical strains (AB133, ABF7, and ABE5) with CRISPR-Cas genes was determined by PCR (primers are shown in Supplementary Table 1).

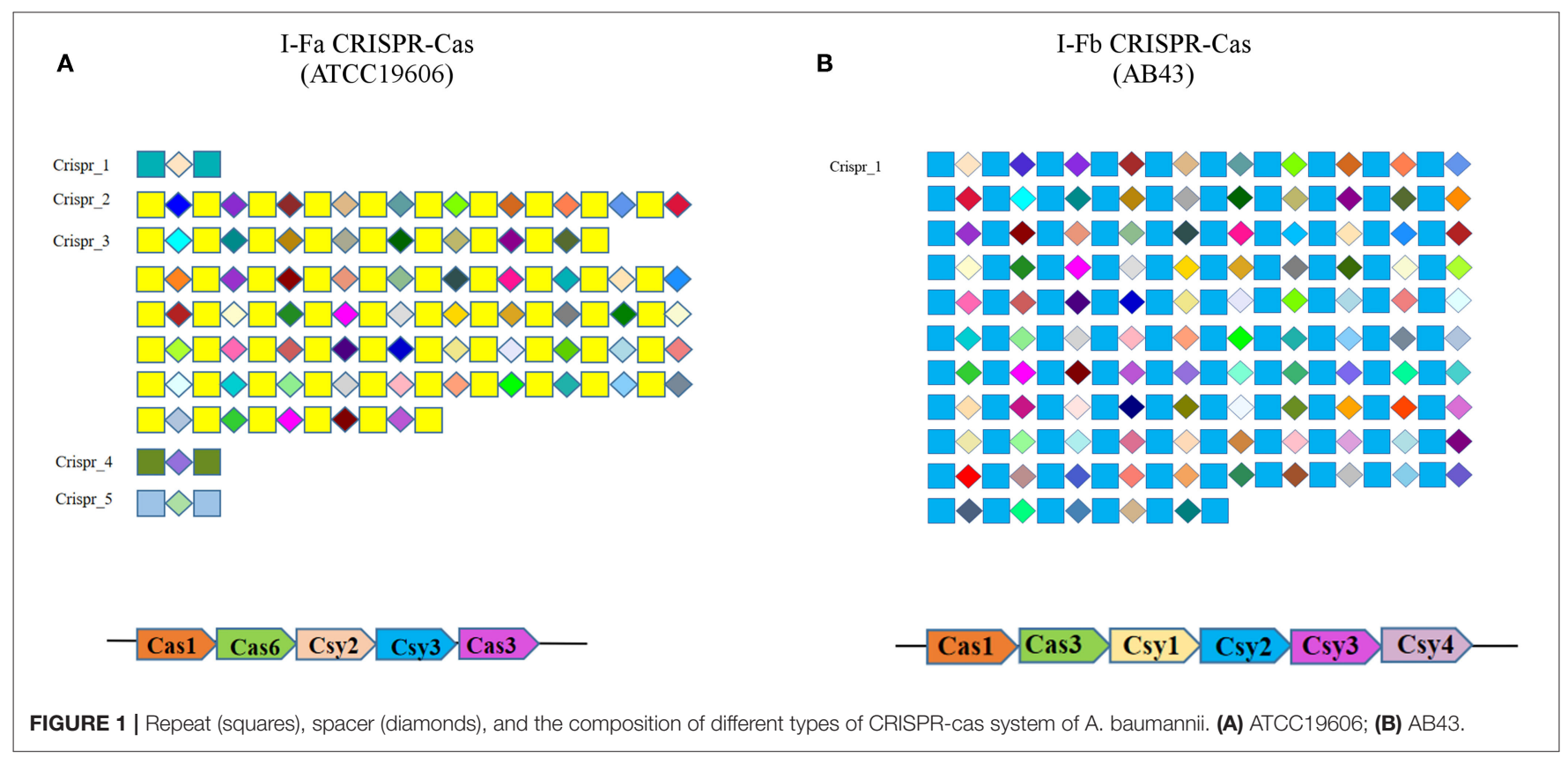




\section{Antimicrobial Susceptibility Assessment}

Strains with different CRISPR-Cas systems were subjected to determine the minimum inhibitory concentrations (MICs) of doxycycline, minocycline, tigecycline, ceftriaxone, imipenem, gentamicin, kanamycin, ciprofloxacin, polymyxin B, colistin, erythromycin, and rifampin to detect the relationship of CRISPRCas system integrity with drug resistance. The MICs of antibiotics were measured using the standard broth microdilution method, according to the Clinical and Laboratory Standard Institute 2020 guideline.

\section{Real-Time Quantitative Reverse Transcription-PCR (qRT-PCR) to Detect CRISPR Gene Expression After Exposure to Antimicrobials}

Strains ATCC19606 and AB43 were grown to the earlyexponential phase and incubated with the MICs of antibiotics for $4 \mathrm{~h}$. Total RNA was extracted from the bacteria using a total RNA extraction kit (TIANGEN, Beijing, PR China). The HISCRIPT 1st strand cDNA synthesis kit (Vazyme, Nanjing, China) was used to synthesize cDNA. A housekeeping gene (16S rRNA gene) was used as an internal control for each sample. qRT-PCR was performed on an ABI 7,500 RT-PCR system (Applied Biosystems, CA, USA), and SYBR Green was the dye (Vazyme, Nanjing, China) utilized. Primers used for qRTPCR are illustrated in Supplementary Table 2. The relative gene expression levels were compared with those of 16S rRNA by the $2^{-\Delta \Delta C t}$ method.

\section{Construction of the csy1 Gene Deletion Mutant}

To delete the csy1 gene from A. baumannii AB43, pKD4 was used as a template to amplify the complete kanamycin cassette gene fragments. The PCR amplification products were identified by $1 \%$ agarose gel electrophoresis and purified using the FastPure Gel DNA Extraction Mini Kit (Vazyme). A. baumannii carrying RecAb on pMMB67EH (pAT04) was inoculated into liquid Luria Bertani (LB) medium containing carbenicillin to preserve the plasmid. IPTG was added to bacteria to the mid$\log$ phase with a final concentration of $2 \mathrm{mM}$, incubated at $37^{\circ} \mathrm{C}$ for $3 \mathrm{~h}$. Then bacteria were collected, washed thrice with $10 \%$ ice-cold glycerol, 1,000-fold concentrated. One microgram recombinant engineering PCR products were electrotransformed into A. baumannii AB43 competent cells $\left(100 \mu \mathrm{L}, \sim 10^{10}\right.$ bacteria) in $2 \mathrm{~mm}$ cuvette at $1.8 \mathrm{kV}$. The bacteria are grown in a $4 \mathrm{~mL}$ rich medium containing $2 \mathrm{mM}$ IPTG for $4 \mathrm{~h}$, centrifuged at 4,000 rpm for $10 \mathrm{~min}$, and removed the supernatant, added in $100 \mu \mathrm{L} \mathrm{LB}$. Then, positive clones were selected from LB agar medium containing $50 \mu \mathrm{g} / \mathrm{mL}$ kanamycin incubated overnight at $37^{\circ} \mathrm{C}$. Screening primers outside the homology region were used to confirm the insertion of the kanamycin cassette (20). Then the following steps were made to make the kanamycin cassette lost in the AB43 $\Delta$ csy1::kan mutant strains. First, pAT03 (pMMB67EH with flippase recombinase) was electrotransformed into AB43 $\Delta$ csy1::kan mutant strains. Then, the bacteria were resuscitated and cultured using LB with $2 \mathrm{mM}$ IPTG for $1 \mathrm{~h}$. Positive clones were then selected on LB agar plates containing carbenicillin $(75 \mu \mathrm{g} / \mathrm{mL})$ incubated overnight at $37^{\circ} \mathrm{C}$. The csy 1 gene knock-out mutation was confirmed by PCR and DNA sequencing (Tsingke Biotechnology Co., China).

\section{Complementation of $\Delta$ csy 1 Mutant Strain}

The pMMB67EH vector was utilized to generate a csy1 gene complementation vector. An 1,110 bp fragment containing an open reading frame of csyl gene from the genome was amplified and ligated to the pMMB67EH vector, then the recombinant plasmid was electrotransformed into the $\mathrm{AB} 43 \Delta c s y 1$. Complementation vector-transformed $\Delta c s y 1$ mutants were selected on LB agar plates containing $50 \mu \mathrm{g} / \mathrm{mL}$ kanamycin and $10 \mu \mathrm{g} / \mathrm{mL}$ tetracycline. Positive clones were verified by colony PCR using primers and DNA sequencing (Tsingke Biotechnology Co, China). The expression of the csy1 gene in $\mathrm{AB} 43$, complemented mutant, and $\Delta c s y 1$ mutant strains was determined by qRT-PCR as described above.

\section{Antimicrobial Susceptibility Testing of Mutant Strains and Resistance Development Studies}

For in vitro resistance development of $\mathrm{AB} 43, \mathrm{AB} 43 \Delta c s y 1 / p c s y 1$, and $A B 43 \Delta c s y 1$, antibiotics sensitive to all three strains were

TABLE 1 | Strains and plasmids used in this study.

\begin{tabular}{|c|c|c|}
\hline Strain/plasmid & Relevant genotype and property & Source and/or reference \\
\hline A. baumannii strain19606 & I-Fa CRISPR-Cas & ATCC \\
\hline A. baumannii strain $\mathrm{AB} 43$ & I-F b CRISPR-Cas & This study \\
\hline A. baumannii strain AB133 & I-F a CRISPR-Cas & This study \\
\hline A. baumannii strain ABF7 & I-F b CRISPR-Cas & This study \\
\hline A. baumannii strain ABE5 & Lack CRISPR-Cas & This study \\
\hline A. baumannii strain $\mathrm{AB} 43 \Delta \mathrm{csy} 1$ & $\Delta c s y 1$ & This study \\
\hline A. baumannii strain AB43 $\Delta$ csy $1 / p c s y 1$ & $\Delta \operatorname{csy} 1 /$ pcsy 1 & This study \\
\hline pMMB67EH & $\mathrm{AMP}^{r}$ & This study \\
\hline pAT04 & pMMB67EH with RecAb system, Tet ${ }^{r}$ & This study \\
\hline pAT03 & pMMB67EH with FLP recombinase kan ${ }^{r}$ & This study \\
\hline pKD4 & $\mathrm{Kan}^{r}$ & This study \\
\hline
\end{tabular}

$r$, represents resistance. 
selected, and then resistance development experiments were conducted. Strains were exposed separately to polymyxin B and rifampin for step-wise selection. Strains at the exponential phase were diluted 1: 1,000 into fresh MHB medium supplement with $0.5 \times$ MIC or $0.25 \times$ MIC of polymyxin B and rifampin. After being cultured at $37^{\circ} \mathrm{C}$ for $24 \mathrm{~h}$ at $200 \mathrm{rpm}$, the MIC of each drug was determined by broth microdilution as mentioned before. The process was repeated for 10 generations. Moreover, the ratio of the MIC obtained from every generation to the MIC at the first generation (first contact) was determined. The data are expressed as the relative increase of MIC per generation (21).

\section{Transcriptomic Analysis}

The RNA-Seq analysis of AB43 and AB43 $\Delta$ csy1 was performed as proposed by Kesavan et al. (22). Total RNAs of AB43 and $\mathrm{AB} 43 \Delta$ csy 1 were extracted from cell cultures at the log phase using an RNA extraction kit (TIANGEN, Beijing, PR China) and quantified using a Nanodrop spectrophotometer (Thermo Scientific, Waltham, MA, USA) by the ratio of absorbance $(260 \mathrm{~nm} / 280 \mathrm{~nm})$. cDNA synthesis, library generation, and data analysis were performed by Shanghai Sheng gong Bioengineering Company, and transcriptome sequencing was conducted using Illumina HiSeqTM.

\section{RESULTS}

\section{Spacer Identification and CRISPR Analysis of $A$. baumannii}

CRISPR-Cas Finder was used to determine the number and sequence of spacers in the CRISPR-Cas repeat array of ATCC19606 and AB43. The results are illustrated in Figure 1. The results demonstrate that ATCC19606 carries five CRISPRs (Crispr_1, Crispr_2, Crispr_3, Crispr_4, and Crispr_5), where the number of spacers in the CRISPR locus of Crispr_1, Crispr_2, Crispr_3, Crispr_4, and Crispr_5 is 1, 18, 45, 1, and 1, respectively. The Type I-Fa Cas cluster consists of Cas1, Cas6, Csy3, Csy2, and Cas3-Cas2 (Cas3). Strain AB43 carries one confirmed CRISPR, which contains 105 spacers, and the Type I-Fb Cas cluster contains Cas1, Cas3-Cas2 (Cas3), Csy1, Csy2, Csy3, and Csy4.

\section{Antimicrobial Susceptibility of Selected Strains}

The status of the CRISPR-Cas system in selected A. baumannii strains is demonstrated in Table 2. To investigate the relationship between csy 1 gene and drug resistance, the relationship between the CRISPR-Cas carrier rate and drug resistance was first investigated. The strains with a complete CRISPR-Cas system (ATCC19606 and AB43) were sensitive to 12 antibiotics tested. However, strains with an incomplete set of CRISPR-Cas-related genes (A133 and F7) or without the CRISPR-Cas system (E5) were resistant to most of the antibiotics tested. The results are shown in Table 3. Thus, the CRISPR-Cas system was speculated to be related to the drug resistance of $A$. baumannii.

\section{CRISPR-Cas Gene Expression in ATCC19606 and AB43 Exposed to Antibiotics}

qRT-PCR analysis of CRISPR-Cas-related cas and csy genes was performed to further study the relationship between the CRISPRCas system and antimicrobial resistance. The expression of the CRISPR-Cas-related genes cas and csy changed under antibiotic pressure (Figures 2A,B). Downregulation of cas1, cas3, cas6, csy2, csy3, and csy4 was detected in ATCC19606 and AB43 when treated with most antibiotics. However, csy1 gene in AB43 was upregulated when treated with most antibiotics, and only downregulated when treated with doxycycline and kanamycin.

TABLE 3 | Minimum inhibitory concentrations (MICs) of strains with different types of CRISPR-Cas system.

\begin{tabular}{lccccc}
\hline Drugs $(\boldsymbol{\mu} \mathbf{g} / \mathbf{m l})$ & ATCC19606 & AB43 & A133 & F7 & E5 \\
\hline Doxycycline & $0.125(\mathrm{~S})$ & $0.125(\mathrm{~S})$ & $64(\mathrm{R})$ & $32(\mathrm{R})$ & $32(\mathrm{R})$ \\
Minocycline & $0.0624(\mathrm{~S})$ & $0.015625(\mathrm{~S})$ & $32(\mathrm{R})$ & $8(\mathrm{l})$ & $2(\mathrm{~S})$ \\
Tigecycline & $2(\mathrm{~S})$ & $0.5(\mathrm{~S})$ & $64(\mathrm{R})$ & $8(\mathrm{l})$ & $8(\mathrm{l})$ \\
Ceftriaxone & $8(\mathrm{~S})$ & $2(\mathrm{~S})$ & $\geq 8192(\mathrm{R})$ & $256(\mathrm{R})$ & $1024(\mathrm{R})$ \\
Imipenem & $8(\mathrm{~S})$ & $8(\mathrm{~S})$ & $\geq 512(\mathrm{R})$ & $512(\mathrm{R})$ & $\geq 512(\mathrm{R})$ \\
Gentamicin & $8(\mathrm{~S})$ & $0.5(\mathrm{~S})$ & $1024(\mathrm{R})$ & $\geq 8192(\mathrm{R})$ & $\geq 8192(\mathrm{R})$ \\
Kanamycin & $8(\mathrm{~S})$ & $4(\mathrm{~S})$ & $\geq 8192(\mathrm{R})$ & $\geq 8192(\mathrm{R})$ & $\geq 8192(\mathrm{R})$ \\
Ciprofloxacin & $0.25(\mathrm{~S})$ & $1(\mathrm{~S})$ & $256(\mathrm{R})$ & $16(\mathrm{R})$ & $32(\mathrm{R})$ \\
Polymyxin B & $0.25(\mathrm{~S})$ & $0.125(\mathrm{~S})$ & $2(\mathrm{~S})$ & $2(\mathrm{~S})$ & $2(\mathrm{~S})$ \\
Colistin & $0.125(\mathrm{~S})$ & $0.125(\mathrm{~S})$ & $0.25(\mathrm{~S})$ & $0.125(\mathrm{~S})$ & $0.125(\mathrm{~S})$ \\
Erythromycin & $8(\mathrm{~S})$ & $1(\mathrm{~S})$ & $256(\mathrm{R})$ & $1(\mathrm{~S})$ & $4(\mathrm{~S})$ \\
Rifampin & $1(\mathrm{~S})$ & $0.5(\mathrm{~S})$ & $64(\mathrm{R})$ & $2(\mathrm{~S})$ & $2(\mathrm{~S})$ \\
\hline
\end{tabular}

$R$ represents resistance; S represents sensitivity.

TABLE 2 | Strains with different carrying situations of CRISPR-Cas system genes.

\begin{tabular}{|c|c|c|c|c|c|c|c|c|}
\hline Strain & ST & cas 1 & cas3 & cas 6 & csy1 & csy2 & csy3 & csy4 \\
\hline ATCC19606 (I-Fa) & ST931 & + & + & + & - & + & + & - \\
\hline AB43 (I-Fb) & ST705 & + & + & - & + & + & + & + \\
\hline AB133 (I-Fa) & ST1145 & + & - & - & - & + & - & - \\
\hline ABF7 (I-Fb) & ST195 & + & - & + & + & + & + & + \\
\hline E5 & ST195 & - & - & - & - & - & - & - \\
\hline
\end{tabular}

+, contain; -, lack. 
A

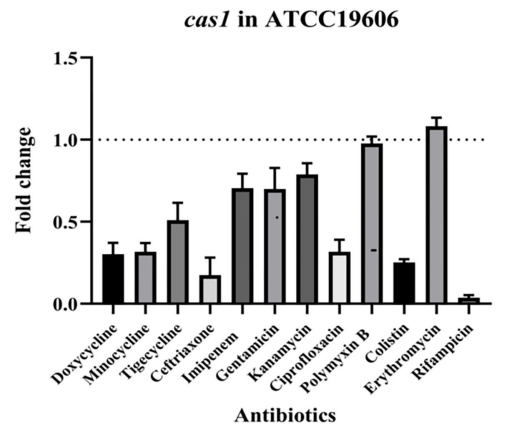

csy2 in ATCC19606

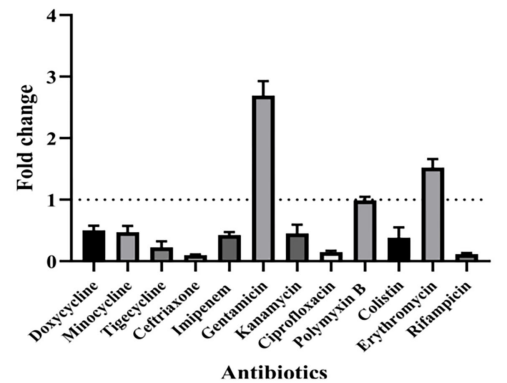

B

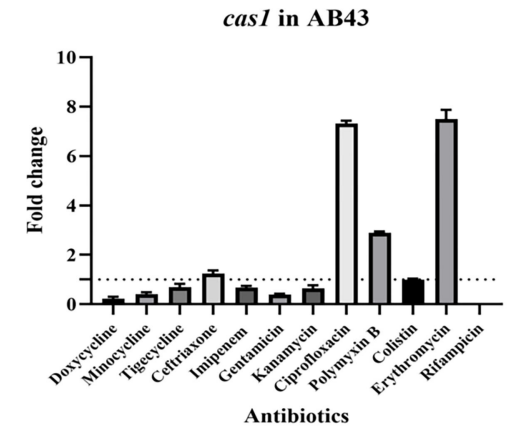

csy2 in AB43

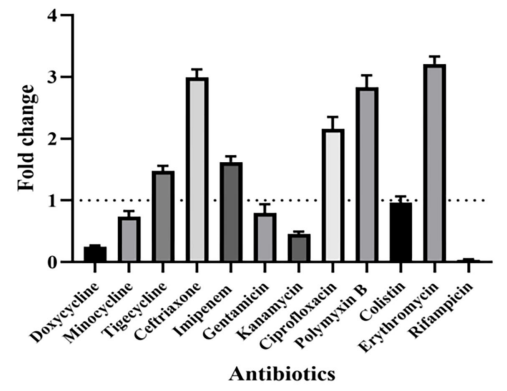

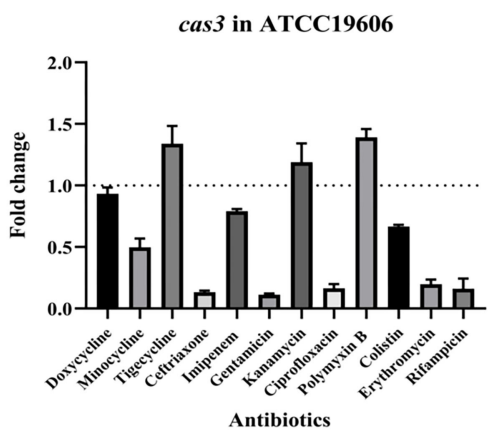

csy3 in ATCC19606

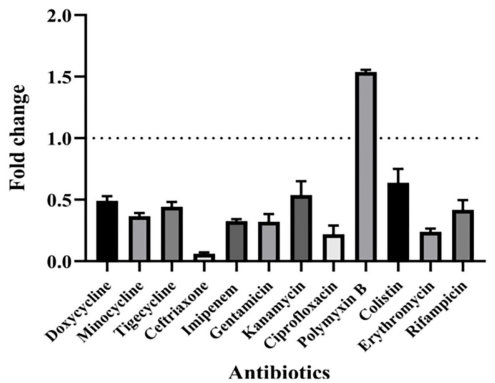

cas3 in AB43

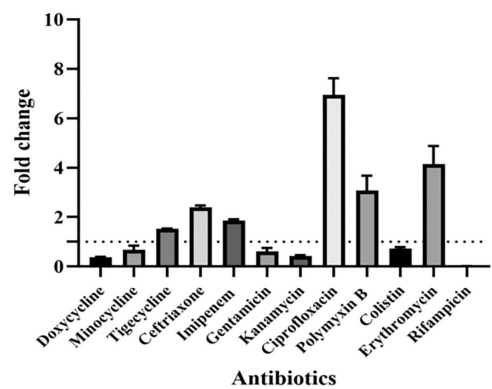

csy3 in $\mathrm{AB} 43$

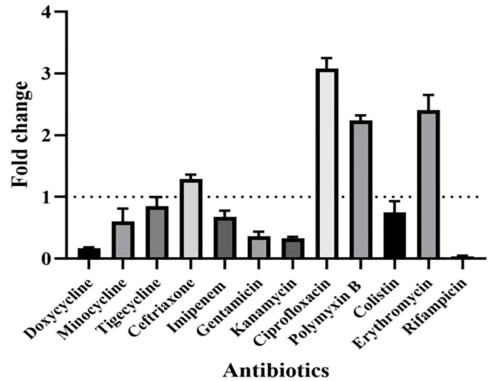

cas6 in ATCC19606

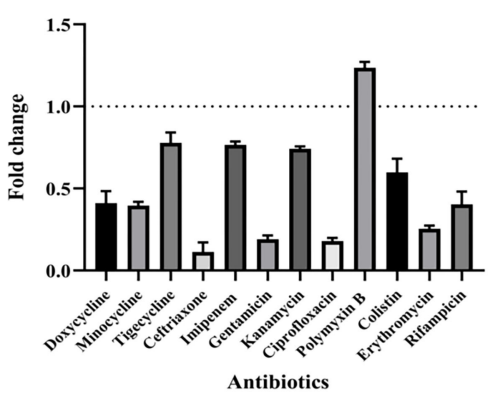

Antibiotics

csy1 in AB43

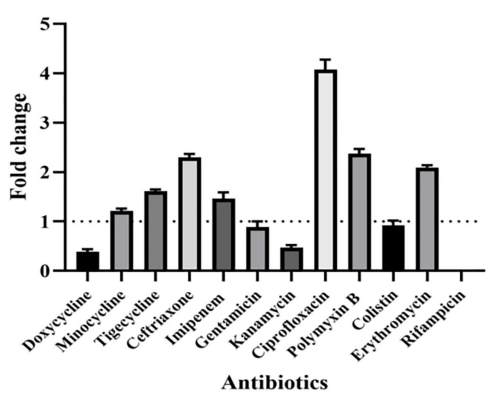

csy4 in AB43

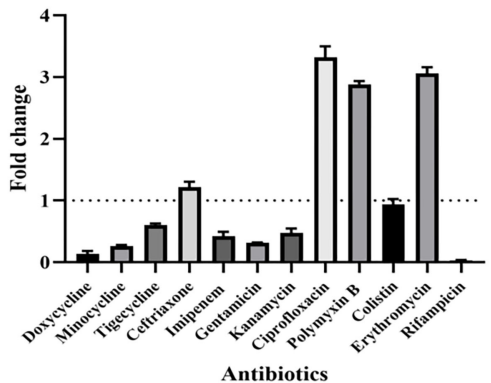

FIGURE 2 | CRISPR-cas gene expression in strains after exposure to antibiotics. (A) CRISPR-related genes (cas1, cas3, cas6, csy2, and csy3) expression in ATCC19606 against antimicrobials; (B) CRISPR-related genes (cas1, cas3, csy1, csy2, csy3, and csy4) expression against antimicrobials in AB43. 


\section{csyl gene expression}

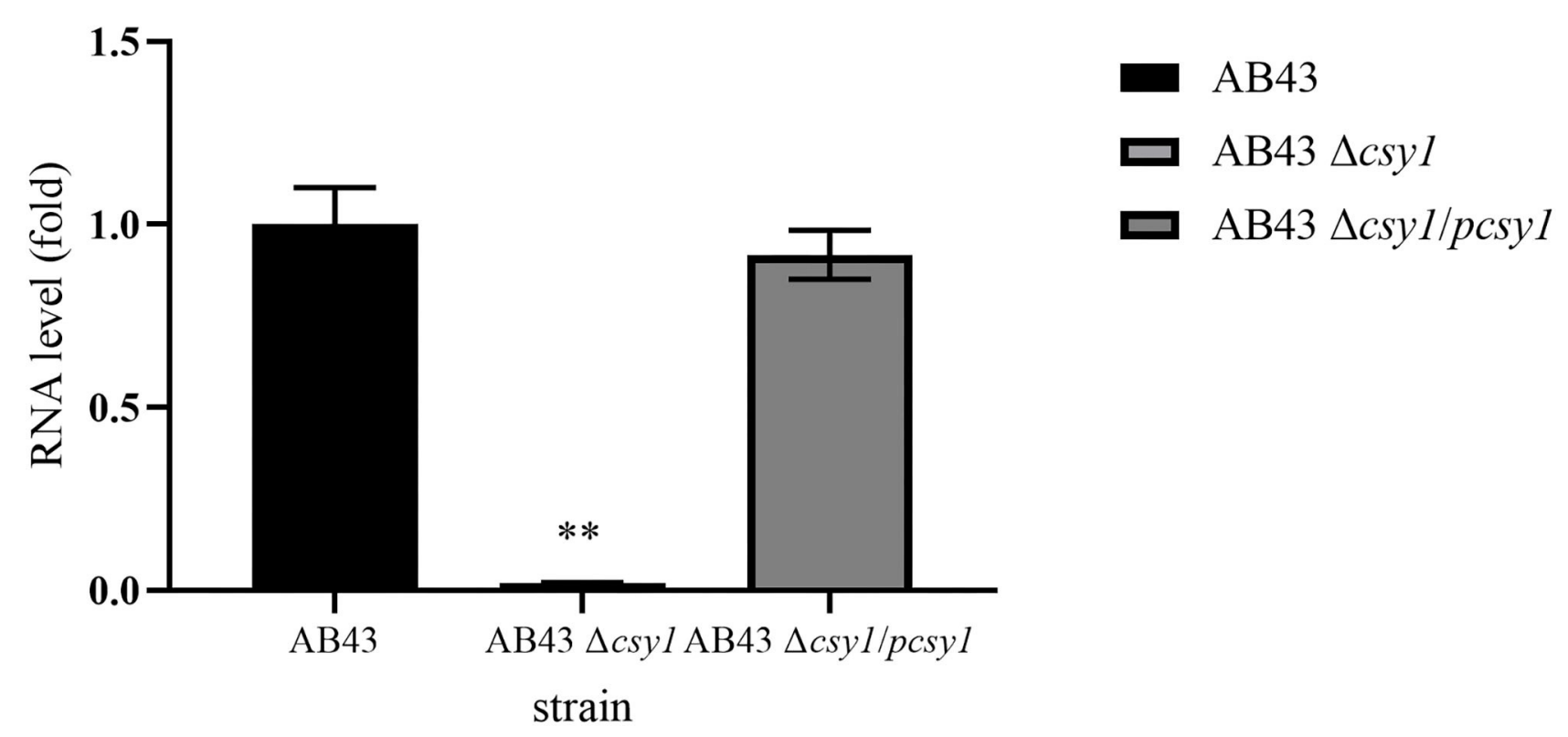

FIGURE 3 | The RNA level of csy1 gene in wild type (AB43), csy1 gene deletion (AB43 $\Delta$ csy1), csy1gene complementation (AB43 $\Delta$ csy1/pcsy1) strains. The RNA level in $A B 43$ was set as 1 , and those in strains $A B 43 \Delta c s y 1$ and $A B 43 \Delta c s y 1 / p c s y 1$ were calculated accordingly. Data are presented as means \pm SEM from three independent experiments. ${ }^{\star *} p<0.01$.

\section{The Role of csy1 Gene in Antimicrobial Susceptibility}

The above studies demonstrated that an integrated CRISPRCas system might inhibit the drug resistance of $A$. baumannii. Further, when A. baumannii was exposed to tested antibiotics, only csyl gene was upregulated. However, little is known regarding the role of csyl gene in the drug resistance of $A$. baumannii. A csy1 gene knock-out strain was constructed. The expressions of csy1 gene RNA in the wild type (AB43), csy1 gene deletion $(\mathrm{AB} 43 \Delta c s y 1)$, and $c s y 1$ gene complementation $(\mathrm{AB} 43 \Delta \operatorname{csy} 1 / \mathrm{pcs} y 1)$ strains were determined by qRT-PCR. The expression of csyl gene RNA was significantly decreased by deletion in the csy1 gene deletion mutant, and restored to wildtype levels in the csyl gene complementation strain (Figure 3). The antimicrobial susceptibility of AB43, the complemented mutant, and $\Delta c s y 1$ mutant strains to 12 drugs was evaluated. The results are shown in Table 4. A 16- to 512-fold (doxycycline, tigecycline, ceftriaxone, imipenem, ciprofloxacin, erythromycin) increase in susceptibility was observed in the $\Delta c s y 1$ mutant strain compared with its wild (AB43) strain. Moreover, a >1,024-fold increase of MIC (minocycline, gentamicin, and kanamycin) was observed in the $\Delta c s y 1$ mutant strain compared with $\mathrm{AB} 43$. The MICs of polymyxin B, colistin, and rifampin were similar in the three strains.

\section{The Role of csy1 Gene in Resistance Development}

Based on the results that csyl gene can regulate the drug resistance of $A$. baumannii, a drug resistance development
TABLE 4 | Minimum inhibitory concentrations (MICs) of AB43, complemented mutant, and $\Delta c s y 1$ mutant strains to different antibiotics.

\begin{tabular}{|c|c|c|c|}
\hline Drugs $(\mu \mathrm{g} / \mathrm{ml})$ & AB43 & AB43 $\Delta$ csy $1 /$ pcsy 1 & $\mathrm{AB} 43 \Delta c s y 1$ \\
\hline Doxycycline & $0.125(\mathrm{~S})$ & $0.015625(\mathrm{~S})$ & $16(\mathrm{R})$ \\
\hline Minocycline & $0.015625(\mathrm{~S})$ & $0.015625(\mathrm{~S})$ & $16(\mathrm{R})$ \\
\hline Tigecycline & $0.5(\mathrm{~S})$ & $8(\mathrm{~S})$ & $128(R)$ \\
\hline Ceftriaxone & $2(S)$ & $4(S)$ & 256(R) \\
\hline Imipenem & $8(S)$ & $8(\mathrm{~S})$ & $\geq 4096(R)$ \\
\hline Gentamicin & $0.5(\mathrm{~S})$ & $1(\mathrm{~S})$ & $\geq 4096(\mathrm{R})$ \\
\hline Kanamycin & $4(S)$ & $4(S)$ & $\geq 4096(\mathrm{R})$ \\
\hline Ciprofloxacin & $1(\mathrm{~S})$ & $0.0625(\mathrm{~S})$ & $16(\mathrm{R})$ \\
\hline Erythromycin & $1(\mathrm{~S})$ & $0.5(\mathrm{~S})$ & $64(\mathrm{R})$ \\
\hline Colistin & $0.125(\mathrm{~S})$ & $0.0625(\mathrm{~S})$ & $0.25(\mathrm{~S})$ \\
\hline Polymyxin B & $0.125(S)$ & $0.125(S)$ & $0.125(S)$ \\
\hline Rifampin & $0.5(\mathrm{~S})$ & $0.5(\mathrm{~S})$ & $1(\mathrm{~S})$ \\
\hline
\end{tabular}

$R$ represents resistance; $S$ represents sensitivity.

assay was done to further elucidate its role in antibiotic resistance. No significant differences were found between AB43, $\mathrm{AB} 43 \Delta \operatorname{cs} y 1 / \mathrm{p} c s y 1$, and $\mathrm{AB} 43 \Delta \operatorname{cs} y 1$ in resistance to polymyxin $\mathrm{B}$ and rifampin (Table 4); thus, both were chosen for the resistance induction experiment. There was no significant difference among $\mathrm{AB} 43, \mathrm{AB} 43 \Delta c s y 1 / p c s y 1$, and $\mathrm{AB} 43 \Delta c s y 1$ in resistance to polymyxin B until the sixth step of selection $(2 \mu \mathrm{g} / \mathrm{mL})$. At $2 \mu \mathrm{g} / \mathrm{mL}$ polymyxin $\mathrm{B}$, only $\mathrm{AB} 43 \Delta c s y 1$ exhibited growth (Figure 4). The drug resistance of rifampicin showed a difference at the third selection step and a 16-fold increase at the eighth 
A

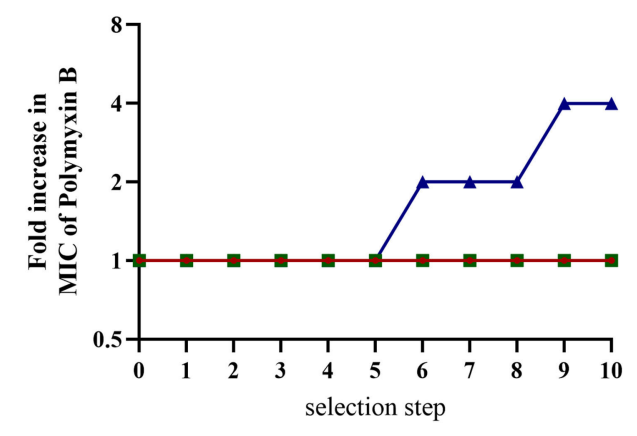

B

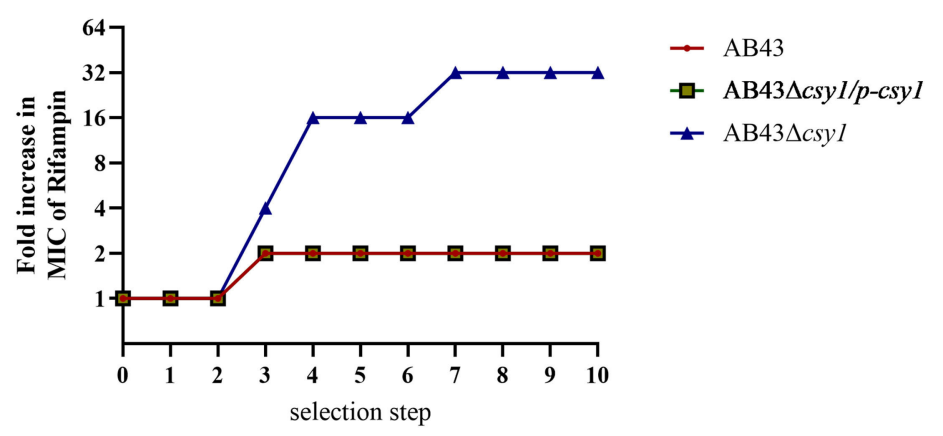

FIGURE 4 | Polymyxin B and rifampicin resistance gains during continuous passaging. (A) Polymyxin B; (B) Rifampicin.

selection step (Figure 4). The results suggested that the csyl gene mutant strain drug resistance developed significantly faster than that of the wild strain.

\section{Transcriptomic Analysis of the csy 1 Gene Knock-Out Mutant}

Transcriptome analyses of AB43 and AB43 $\Delta$ csy1 were performed, which demonstrated that genes encoding efflux protein, the $50 \mathrm{~S} / 30 \mathrm{~S}$ ribosomal proteins, proteins involved in energy metabolism, and cytochrome $\mathrm{C}$ were upregulated in $A B 43 \Delta c s y 1$ (Figure 5). The upregulation of the gene encoding the efflux pump was reported to be associated with increased antimicrobial resistance (23). Additionally, the increased synthesis of ATP could promote drug efflux. Several downregulated genes in the mutant strain were also detected. After the csy 1 gene was knocked out, the expression of CRISPRCas-related genes was downregulated. The related proteins of the CRISPR-Cas system were speculated to influence each other as a whole. The decreased expression of membrane-related proteins indicated that the CRISPR-Cas system might regulate cell membrane formation.

\section{DISCUSSION}

The primary role of the CRISPR-Cas system is bacterial defense against phages, plasmids, and other foreign DNA. Some recent studies showed that the CRISPR-Cas system might play a role in antibiotic resistance of bacteria (24). The effect of the CRISPRCas system on antibiotic resistance varies in different bacteria. The CRISPR-Cas system promotes resistance in C. jejuni and inhibits resistance in E. coli and E. fecium $(8,9,25)$. However, the relationship of the CRISPR-Cas system with antibiotic resistance in A. baumannii is not fully elucidated. By investigating the carrying situation of the CRISPR-Cas system and the drug resistance level of selected strains, we assessed whether the CRISPR-Cas system plays a role in A. baumannii antibiotic resistance. The results showed that the complete CRISPR-Cas system might inhibit bacterial drug resistance. qRT-PCR analysis was conducted to measure the expression levels of Type I-Fa and I-Fb CRISPR-Cas system-related genes after antibiotic treatment. The results demonstrated that the expressions of cas and csy genes were modified.

With the excessive use of antibiotics, the bacterial resistance rate has continued to increase. The infection rate of MDR pathogens is increasing. Thus, the development of different strategies to combat antimicrobial resistance is critical (4). Moreover, research on the relationship between CRISPR-Cas and bacterial resistance is primarily focused on the cas 3 and cas9 genes (26). The cas3 gene in Streptococcus mutants can regulate biofilm formation and the ability to resist fluoride (27). Sampson et al. proved that the CRISPR-Cas endonuclease gene cas 9 together with tracrRNA and ScaRNA is essential for enhancing the stability of the bacterial envelope and promoting antibiotic resistance (10). However, the relationship between the csy 1 gene and drug resistance in bacteria has not been previously reported. To the best of our knowledge, this is the first study regarding the relationship between csyl gene and drug resistance in A. baumannii. Preliminary studies have demonstrated that csy 1 gene can regulate the drug resistance of $A$. baumannii and maintain its sensitivity to antibiotics. After knocking out the csyl gene, the bacterial resistance level increased. Simultaneously, the drug resistance experiment showed that csy1 gene could inhibit the drug resistance development of bacteria. The complete CRISPR-Cas system may inhibit its drug resistance. The results are similar to studies on Enterococcus fecalis, which found that the lack of CRISPR genes is related to species, multidrug resistance, and major drug resistance-related genes (28).

The study by Aydin et al. suggested that the CRISPR-Cas system may interfere with the acquisition of resistant plasmids, thereby maintaining the sensitivity of these strains (9). Klebsiella pneumoniae strains detected that the spacers in the CRISPR array matched the genome of the plasmid or phage, some containing resistance genes (29). In Francis bacteria, CRISPR-Cas can enhance the integrity of its envelope, leading to resistance to several membrane stressors, including antibiotics, and increasing 
A

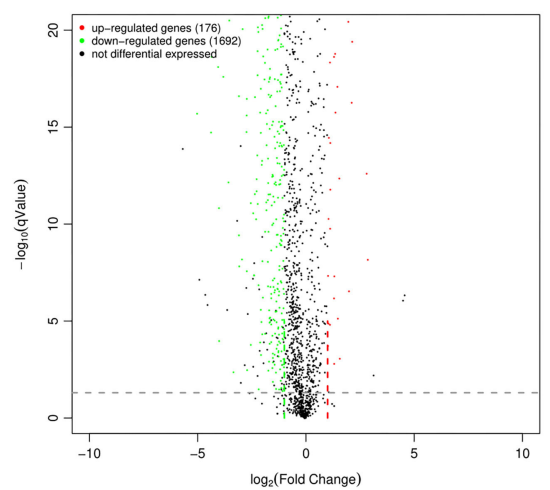

B

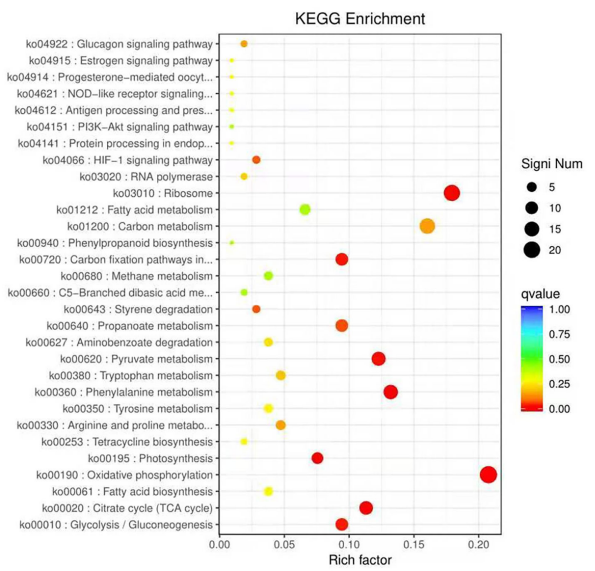

D
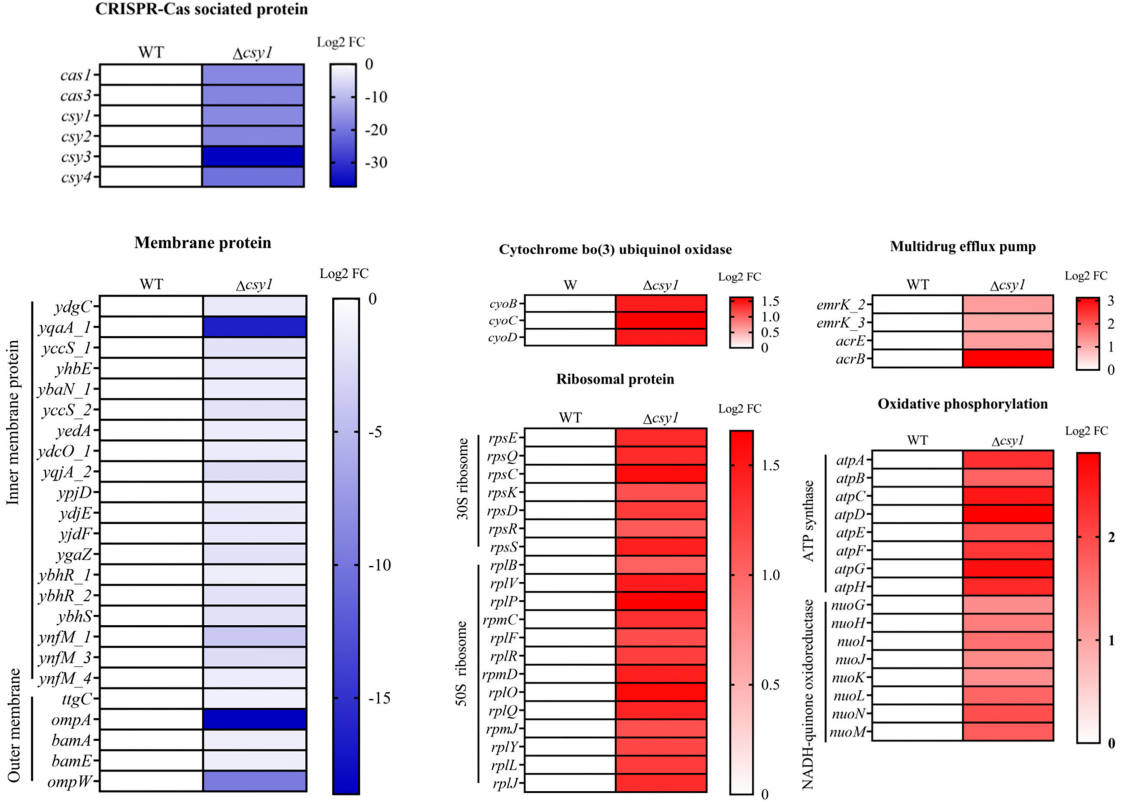

FIGURE 5 | Transcriptome analysis of AB43 and AB43 $\triangle$ csy1. (A) Differential gene volcano map; (B) Kyoto Encyclopedia of Genes and Genomes (KEGG) enrichment analysis of up-regulation genes; (C) KEGG enrichment analysis of down-regulation genes; the color of the dot represents the size of the Q-value, and the size of the dot represents the number of differential genes; (D) Selected differential expression genes involved in CRISPR-Cas related protein, ribosome protein, oxidative phosphorylation related protein, a membrane-associated related protein, and multidrug efflux pump protein. 
antibiotic resistance (10). Transcriptome analysis was conducted to assess the mechanism of csyl gene inhibiting the drug resistance of $A$. baumannii. The csyl gene mutant strain has a higher expression of drug efflux pump, ATP synthesis-related, and ribosomal genes, as well as a lower expression of CRISPRCas-related and membrane-related genes.

Thus, with the steady increase in MDR bacterial infections, the innovation of novel therapies to combat MDR bacteria is critical. Existing studies have demonstrated that the CRISPR-Cas system delivered by phages can sequentially eliminate Staphylococcus aureus (30). CRISPR-encoded presentation plasmids or CRISPRCas antibacterial drugs can reduce the occurrence of antibiotic resistance in Enterococci (31). The delivery of CRISPR-Cas9 effectively removes antibiotic resistance in vitro by targeting plasmid-borne resistance genes (32). Our work main revealed that the $c s y 1$ gene participates in the regulation of drug resistance in A. baumannii. The present study has several limitations. It is unknown how csyl gene, regulates drug resistance in A. baumannii. Moreover, we only performed csyl gene knock-out experiments in AB43 (I-Fb CRISPR-Cas), and more strains are needed to generalize our results. Thus, future studies should focus on assessing the mechanism by which CRISPR-Cas regulates drug resistance. Moreover, deploying the Type I CRISPR-Cas system as an antimicrobial to treat drug-resistant $A$. baumannii infection is an attractive strategy compared with conventional antibiotic therapy. It is also necessary to explore the effect of other CRISPRCas components in modulating aspects of bacterial physiology such as virulence and drug resistance.

\section{CONCLUSIONS}

In conclusion, we studied the relationship of the CRISPR-Cas system with antibiotic resistance in A. baumannii. Our results revealed that the expression of CRISPR-Cas related genes was changed under antibiotic pressure and that the presence of the csyl gene has an inhibitory effect on the drug resistance of A. baumannii. Deletion of the csy1 gene in A. baumannii strain AB43 made it resistant to most of the antibiotics tested. In addition, this study extends our understanding of resistance regulation of $A$. baumannii and provides a new direction for studying the functions of CRISPR-Cas systems in drug resistance.

\section{REFERENCES}

1. Sarshar M, Behzadi P, Scribano D, Palamara AT, Ambrosi C. Acinetobacter baumannii: an ancient commensal with weapons of a pathogen. Pathogens. (2021) 10:387. doi: 10.3390/pathogens10040387

2. Liang C, Zhang X, Zhou L, Meng G, Zhong L, Peng P. Trends and correlation between antibacterial consumption and carbapenem resistance in gramnegative bacteria in a tertiary hospital in China from 2012 to 2019. BMC Infect Dis. (2021) 21:444. doi: 10.1186/s12879-021-06140-5

3. Kyriakidis I, Vasileiou E, Pana ZD, Tragiannidis A. Acinetobacter baumannii antibiotic resistance mechanisms. Pathogens. (2021) 10:373. doi: 10.3390/pathogens 10030373

4. Shabbir MAB, Shabbir MZ, Wu Q, Mahmood S, Sajid A, Maan MK, et al. CRISPR-cas system: biological function in microbes and its use to treat
Future work can be focused on their functions that extend beyond the general disruption of invading foreign DNA and may clarify how CRISPR-Cas systems contribute to bacterial resistance and virulence. This knowledge will be useful in the exploration of new antibacterial strategies.

\section{DATA AVAILABILITY STATEMENT}

The datasets presented in this study can be found in online repositories. The names of the repository/repositories and accession number(s) can be found at: NCBI SRA; SRR17253291 and SRR17253292.

\section{AUTHOR CONTRIBUTIONS}

GL and TG contributed to the design of the study. TG, XS, ML, and YW contributed to the acquisition of the data. GL, TG, and XS contributed to the analysis of the data. All authors contributed to data interpretation, drafting the manuscript, critically revising the manuscript for important intellectual content, and approved the final version of the manuscript.

\section{FUNDING}

This work was supported by the National Natural Science Foundation of China (82073611 and 82002186), the Natural Science Foundation of the Higher Education Institutions of Jiangsu Province (Grant Number: 19KJB310002).

\section{ACKNOWLEDGMENTS}

The authors are grateful for the manuscript correction by Dr. Benjamin Chen from Yale University School of Medicine PET center.

\section{SUPPLEMENTARY MATERIAL}

The Supplementary Material for this article can be found online at: https://www.frontiersin.org/articles/10.3389/fmed. 2022.797104/full\#supplementary-material

antimicrobial resistant pathogens. Ann Clin Microbiol Antimicrob. (2019) 18:21. doi: 10.1186/s12941-019-0317-x

5. Shmakov SA, Makarova KS, Wolf YI, Severinov KV, Koonin EV. Systematic prediction of genes functionally linked to CRISPR-Cas systems by gene neighborhood analysis. Proc Natl Acad Sci USA. (2018) 115:E530716. doi: $10.1073 /$ pnas. 1803440115

6. Price VJ, McBride SW, Hullahalli K, Chatterjee A, Duerkop BA, Palmer KL. Enterococcus faecalis CRISPR-Cas is a robust barrier to conjugative antibiotic resistance dissemination in the murine intestine. mSphere. (2019) 4:e00464-19. doi: 10.1128/mSphere.00464-19

7. Watson BNJ, Staals RHJ, Fineran PC. CRISPR-cas-mediated phage resistance enhances horizontal gene transfer by transduction. mBio. (2018) 9:e02406-17. doi: 10.1128/mBio.024 06-17 
8. Shabbir MA, Wu Q, Shabbir MZ, Sajid A, Ahmed S, Sattar A, et al. The CRISPR-cas system promotes antimicrobial resistance in campylobacter jejuni. Future Microbiol. (2018) 13:1757-74. doi: 10.2217/fmb-20180234

9. Aydin S, Personne Y, Newire E, Laverick R, Russell O, Roberts AP, et al. Presence of Type I-F CRISPR/Cas systems is associated with antimicrobial susceptibility in Escherichia coli. J Antimicrob Chemother. (2017) 72:22138. doi: 10.1093/jac/dkx137

10. Sampson TR, Napier BA, Schroeder MR, Louwen R, Zhao J, Chin CY, et al. A CRISPR-Cas system enhances envelope integrity mediating antibiotic resistance and inflammasome evasion. Proc Natl Acad Sci USA. (2014) 111:11163-8. doi: 10.1073/pnas.1323025111

11. Hong S, Ka D, Yoon SJ, Suh N, Jeong M, Suh JY, et al. CRISPR RNA and anti-CRISPR protein binding to the xanthomonas albilineans Csy1Csy2 heterodimer in the type I-F CRISPR-Cas system. J Biol Chem. (2018) 293:2744-54. doi: 10.1074/jbc.RA117.001611

12. Wiedenheft B, van Duijn E, Bultema JB, Waghmare SP, Zhou K, Barendregt A, et al. RNA-guided complex from a bacterial immune system enhances target recognition through seed sequence interactions. Proc Natl Acad Sci USA. (2011) 108:10092-7. doi: 10.1073/pnas.1102716108

13. Makarova KS, Wolf YI, Iranzo J, Shmakov SA, Alkhnbashi OS, Brouns SJJ, et al. Evolutionary classification of CRISPR-Cas systems: a burst of class 2 and derived variants. Nat Rev Microbiol. (2020) 18:6783. doi: 10.1038/s41579-019-0299-x

14. Fagerlund RD, Wilkinson ME, Klykov O, Barendregt A, Pearce FG, Kieper $\mathrm{SN}$, et al. Spacer capture and integration by a type I-F Cas1-Cas2-3 CRISPR adaptation complex. Proc Natl Acad Sci USA. (2017) 114:E51228. doi: 10.1073/pnas.1618421114

15. Pausch P, Müller-Esparza H, Gleditzsch D, Altegoer F, Randau L, Bange G. Structural variation of type I-F CRISPR RNA guided DNA surveillance. Mol Cell. (2017) 67:622-32.e624. doi: 10.1016/j.molcel.2017.06.036

16. Karah N, Samuelsen O, Zarrilli R, Sahl JW, Wai SN, Uhlin BE. CRISPR-cas subtype I-Fb in acinetobacter baumannii: evolution and utilization for strain subtyping. PLoS ONE. (2015) 10:e0118205. doi: 10.1371/journal.pone.0118205

17. Touchon M, Cury J, Yoon EJ, Krizova L, Cerqueira GC, Murphy C, et al. The genomic diversification of the whole acinetobacter genus: origins, mechanisms, and consequences. Genome Biol Evol. (2014) 6:286682. doi: 10.1093/gbe/evu225

18. Tyumentseva M, Mikhaylova Y, Prelovskaya A, Tyumentsev A, Petrova L, Fomina V, et al. Genomic and phenotypic analysis of multidrugresistant acinetobacter baumannii clinical isolates carrying different types of CRISPR/Cas systems. Pathogens. (2021) 10:205. doi: 10.3390/pathogens1002 0205

19. Zheng Y, Li J, Wang B, Han J, Hao Y, Wang S, et al. Endogenous type I CRISPR-cas: from foreign DNA defense to prokaryotic engineering. Front Bioeng Biotechnol. (2020) 8:62. doi: 10.3389/fbioe.2020.00062

20. Tucker AT, Nowicki EM, Boll JM, Knauf GA, Burdis NC, Trent MS, et al. Defining gene-phenotype relationships in acinetobacter baumannii through one-step chromosomal gene inactivation. mBio. (2014) 5:e013134. doi: $10.1128 / \mathrm{mBio} .01313-14$

21. Maisuria VB, Okshevsky M, Déziel E, Tufenkji N. Proanthocyanidin interferes with intrinsic antibiotic resistance mechanisms of gram-negative bacteria. $A d v$ Sci. (2019) 6:1802333. doi: 10.1002/advs.201802333

22. Kesavan D, Vasudevan A, Wu L, Chen J, Su Z, Wang S, et al. Integrative analysis of outer membrane vesicles proteomics and whole-cell transcriptome analysis of eravacycline induced acinetobacter baumannii strains. BMC Microbiol. (2020) 20:31. doi: 10.1186/s12866-020-1722-1
23. Pontes DS, de Araujo RSA, Dantas N, Scotti L, Scotti MT, de Moura RO, et al. Genetic mechanisms of antibiotic resistance and the role of antibiotic adjuvants. Curr Top Med Chem. (2018) 18:42-74. doi: 10.2174/1568026618666180206095224

24. Roy S, Naha S, Rao A, Basu S. CRISPR-Cas system, antibiotic resistance and virulence in bacteria: through a common lens. Prog Mol Biol Transl Sci. (2021) 178:123-74. doi: 10.1016/bs.pmbts.2020.12.005

25. Sanderson H, Ortega-Polo R, Zaheer R, Goji N, Amoako KK, Brown $\mathrm{RS}$, et al. Comparative genomics of multidrug-resistant enterococcus spp. isolated from wastewater treatment plants. BMC Microbiol. (2020) 20:20. doi: 10.1186/s12866-019-1683-4

26. Vrancianu CO, Gheorghe I, Czobor IB, Chifiriuc MC. Antibiotic resistance profiles, molecular mechanisms and innovative treatment strategies of acinetobacter baumannii. Microorganisms. (2020) 8:935. doi: 10.3390/microorganisms 8060935

27. Tang $\mathrm{B}$, Gong $\mathrm{T}$, Zhou $\mathrm{X}$, Lu $\mathrm{M}$, Zeng J, Peng $\mathrm{X}$, et al. Deletion of cas3 gene in streptococcus mutans affects biofilm formation and increases fluoride sensitivity. Arch Oral Biol. (2019) 99:190-7. doi: 10.1016/j.archoralbio.2019.01.016

28. Dos Santos BA, de Oliveira JDS, Parmanhani-da-Silva BM, Ribeiro RL Teixeira LM, Neves FPG. CRISPR elements and their association with antimicrobial resistance and virulence genes among vancomycin-resistant and vancomycin-susceptible enterococci recovered from human and food sources. Infect Genet Evol. (2020) 80:104183. doi: 10.1016/j.meegid.2020.104183

29. Wang G, Song G, Xu Y. Association of CRISPR/Cas system with the drug resistance in klebsiella pneumoniae. Infect Drug Resist. (2020) 13:192935. doi: 10.2147/IDR.S253380

30. Bikard D, Euler CW, Jiang W, Nussenzweig PM, Goldberg GW, Duportet $\mathrm{X}$, et al. Exploiting CRISPR-Cas nucleases to produce sequence-specific antimicrobials. Nat Biotechnol. (2014) 32:1146-50. doi: 10.1038/nbt.3043

31. Rodrigues M, McBride SW, Hullahalli K, Palmer KL, Duerkop BA. Conjugative delivery of CRISPR-Cas9 for the selective depletion of antibioticresistant enterococci. Antimicrob Agents Chemother. (2019) 63:e0145419. doi: 10.1128/AAC.01454-19

32. Tagliaferri TL, Guimarães NR, Pereira MPM, Vilela LFF, Horz HP, Dos Santos SG, et al. Exploring the potential of CRISPR-Cas9 under challenging conditions: facing high-copy plasmids and counteracting betalactam resistance in clinical strains of enterobacteriaceae. Front Microbiol. (2020) 11:578. doi: 10.3389/fmicb.2020.00578

Conflict of Interest: The authors declare that the research was conducted in the absence of any commercial or financial relationships that could be construed as a potential conflict of interest.

Publisher's Note: All claims expressed in this article are solely those of the authors and do not necessarily represent those of their affiliated organizations, or those of the publisher, the editors and the reviewers. Any product that may be evaluated in this article, or claim that may be made by its manufacturer, is not guaranteed or endorsed by the publisher.

Copyright (c) 2022 Guo, Sun, Li, Wang, Jiao and Li. This is an open-access article distributed under the terms of the Creative Commons Attribution License (CC BY). The use, distribution or reproduction in other forums is permitted, provided the original author(s) and the copyright owner(s) are credited and that the original publication in this journal is cited, in accordance with accepted academic practice. No use, distribution or reproduction is permitted which does not comply with these terms. 